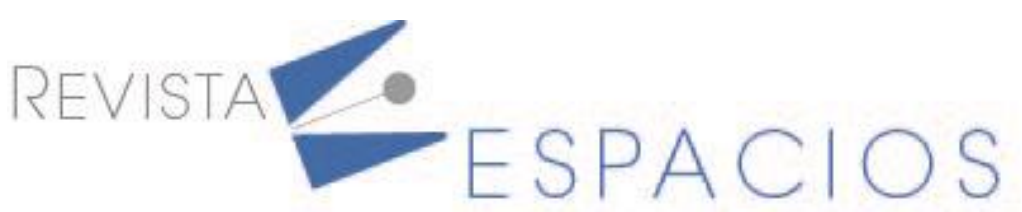

\title{
Mecanismos de transferencia de tecnología y conocimiento en el sector exportador de ganado bovino
}

\section{Mechanisms for technology and knowledge transfer in the cattle export sector}

\author{
MARTELO, Raul J. ${ }^{1}$ \\ LINARES Jose A. ${ }^{2}$ \\ MUÑOZ, Delvis R. ${ }^{3}$
}

\section{Resumen}

El objetivo fue identificar los mecanismos de transferencia de tecnología y conocimiento en las empresas exportadoras de ganado bovino de Barranquilla, Colombia. La investigación es descriptiva y de pronóstico, el diseño es de campo, no experimental transeccional. La población corresponde a especialistas en ganadería del sector exportadora. La técnica de recolección de datos utilizada consiste en la observación mediante encuesta, como instrumento de recolección de datos se emplea un cuestionario bajo una escala tipo Likert. se procedió bajo un análisis estadístico descriptivo. Se manifiesta el mediano interés en implementar otros mecanismos de transferencia, muchos de los cuales tienen cabida en ambientes de vinculación entre el sector académico y el empresarial, donde la transferencia se expresa como la movilización de la tecnología desde los centros generadores del conocimiento hacia las empresas que requieren su uso y explotación, siendo en este caso, una transferencia horizontal o intersectorial.

Palabras clave: ciencia, tecnología, innovación, ganadería.

\begin{abstract}
The objective was to identify determine the technology and knowledge transfer mechanisms in the cattle exporting companies of Barranquilla, Colombia. The research is descriptive and prognostic, the design is field, not experimental, transectional. The population corresponds to livestock specialists in the export sector. The data collection technique used consists of observation through a survey, as a data collection instrument a questionnaire is used under a Likert-type scale. proceeded under a descriptive statistical analysis. The medium interest in implementing other transfer mechanisms is manifested, many of which have a place in environments of linkage between the academic and business sectors, where the transfer is expressed as the mobilization of technology from the centers that generate knowledge to companies. that require its use and exploitation, being in this case, a horizontal or intersectoral transfer.
\end{abstract}

Key words: science, technology, innovation, livestock.

\footnotetext{
${ }^{1}$ Docente de Tiempo Completo. Universidad de Cartagena. Cartagena de indias, Bolivar, Colombia. Email: rmartelog1@unicartagena.edu.co

${ }^{2}$ Docente de Planta. Universidad Politécnica Territorial de Maracaibo. Maracaibo, Zulia, Venezuela. Email: jlinares.proyco@gmail.com

${ }^{3}$ Docente de Tiempo Completo. Universidad de La Guajira. Riohacha, La Guajira, Colombia. Email: dmunozr@uniguajira.edu.co
} 


\section{Introducción}

Para estudiar los mecanismos de transferencia de tecnología y conocimiento, se menciona en primer lugar la clasificación aportada por Casar (2004), quien señala que los instrumentos o mecanismos a través de los cuales se puede llevar a cabo la transferencia de tecnología son los siguientes: convenios y contratos, patentes y licencias, movilidad de recursos humanos, creación de empresas basadas en resultados de investigación universitarias, servicios técnicos y actividades de apoyo a la investigación (Bornachera et al., 2020; Reyes, 2010; Linzer, 2008; Ortuño, 2007).

- Convenios y Contratos: se llevan a cabo para solucionar una necesidad concreta de investigación difícilmente asumible por una organización, bien sea por falta de recursos o de instalaciones. Son los más utilizados para transferir los resultados de investigaciones.

- Patente: es un titulo que reconoce el derecho de explotar en exclusiva la invención patentada, impidiendo a otros su fabricación, venta o utilización sin consentimiento del titular. Ésta se concede a una empresa, a un individuo o a otra entidad desde el momento en que la invención supera los criterios de novedad, actividad inventiva y posibilidad de aplicación industrial.

- Licencias: es un contrato mediante el cual, el titular de la patente o de su solicitud (licenciatante) permite el uso de la misma a un tercero (licenciatario), sin transmitir su titularidad. En términos jurídicos contractuales, denota un negocio jurídico bilateral (contrato) por el cual el titular de un derecho autoriza a otra persona para que ejerza ese derecho, durante determinado tiempo y a cambio de una contraprestación o no.

- Movilidad de recursos humanos: se basa en la movilidad del recurso humano desde los centros de generación de conocimiento hacia el sector productivo. En este sentido, la incorporación de doctores y tecnólogos al ámbito industrial; o la incorporación parcial de científicos en empresas, son dos modalidades en las que el flujo de conocimiento se realiza de forma indirecta mediante el know-how de los expertos.

- Creación de empresas basadas en resultados de investigaciones universitarias (spin-offs): los spin-offs son empresas de base tecnológica cuya idea de negocio surge de los resultados de investigaciones universitarias, y que están gestionadas por el personal propio de la universidad.

- Servicios técnicos y actividades de apoyo a la investigación: se basan en aquellos instrumentos relacionados con la utilización por parte de las empresas de los servicios de apoyo ofrecidos por las universidades, los cuales han mejorado su gestión externa para participar en esta fase de transferencia, tales como algunos servicios científico-tecnológicos y plataformas ligadas a las nuevas tecnologías gestionadas por las universidades o centros de investigación.

De igual manera, se tienen según Lee (2003), diferentes mecanismos de transferencia tecnológica, que pueden ser aplicados entre los centros de investigación de las universidades y la industria: intercambio académico, conferencias y publicaciones; consultorías y provisión de servicios técnicos, Joint Venture de I+D; acuerdos de cooperación de I+D; licenciamiento; parques e incubadoras; entrenamiento y Spin-off (citado por Arenas, 2012; Zarazua et al., 2009).

- Intercambio académico, conferencias, publicaciones: esto es informal y sin costo, el intercambio de información entre universidades, incluye presentación de profesionales y las conferencias técnicas. Esto es ampliamente utilizado, y el primer paso es el enlace entre institutos académicos, centros de investigación y la industria.

- Consultorías y provisión de servicios técnicos: una o más partes de los centros de investigación y universidades, proporcionan un asesoramiento, información o servicios técnicos. Ellos tienen contratos 
formales, generalmente cortos y específicos. Estas pueden ser, de las siguientes formas: Comité de asesoría, Grupos informales de las compañías, y Centros universitarios o unidades de enlace industrial.

- Joint venture de I+D: en el cual se redacta un contrato donde los costos asociados con el trabajo son compartidos y especificados en el contrato. Las dos partes pueden trabajar juntos, a partir de la etapa de la comercialización de I+D. Deben haber mutuos beneficios para la industria y los centros de investigación, y la información de valor comercial, puede ser protegida por un periodo limitado de tiempo.

- Acuerdos de cooperación de I+D: esto es un acuerdo entre uno o más centros de investigación de las universidades y una o más empresas, donde las universidades proveen al personal, instalaciones y otros recursos. Por parte de la industria proporciona fondos, personal, servicios, equipamientos, instalaciones.

- Licenciamiento: es la forma de transferir la tecnología, donde se protege los derechos de la propiedad intelectual. Esto puede ser exclusivo o no. Rogers (2000), menciona como un permiso o los derechos para utilizar cierto producto, diseño o proceso. Esto lleva a presentar las diferentes formas de licencias donde el licenciante, puede otorgar al licenciatario las facultades de la tecnología de las siguientes maneras: licencia exclusiva, licencia única o licencia no exclusiva.

- Contratos de investigación: estos contratos son entre la universidad y la industria. Usualmente las industrias proveen los fondos y la universidad provee la inteligencia. Estos contratos son utilizados por la empresa únicamente para beneficios comerciales.

- Parques científicos, parques de investigación, parques tecnológicos e incubadoras: son instalaciones normalmente creadas por la universidad y con la colaboración de miembros de empresas de alta tecnología, que reciben asistencia en las etapas iníciales. Los principales fondos provienen de los fondos de las empresas participantes.

- Entrenamiento: donde los estudiantes están expuestos a los métodos de trabajo de la industria y los centros de investigación. Muchos centros de investigación, tienen programas de entrenamiento para transferir las tecnologías. Esto puede reducir el riesgo de implementación de la tecnología.

- Spin-off: según Rogers (2000), es una nueva compañía, que está formada de manera individual donde ex trabajadores de una empresa y con el conocimiento de una tecnología transferido de su empresa. Los spin-off, son empresas creadas alrededor de la innovación de una tecnología. Estos pueden clasificarse dependiendo de la empresa desde donde proviene el empleado (Arias \& Segura, 2020; Mango et al., 2016; Pérez, 2003). Existen dos fuentes de mayor influencia, los centros de investigación de instituciones universitarias y las empresas industriales de alta tecnología.

Por su parte, González-Sabater (2011), indica que son diversos los tipos formales que existen para transferir tecnología, sin embargo, todos estos mecanismos "no son utilizables por todos los generadores o usuarios de tecnología, ni sirven para cualquier circunstancia. En ocasiones se combinan varios de ellos en función de las características particulares de la cooperación" (p. 47). Entre ellos se encuentran los acuerdos de licencia; cooperación tecnológica; asistencia técnica y servicios; movilidad de personal; creación de empresas; alianzas tecnológicas; adquisiciones y fusiones; y la compra/venta de bienes y equipos.

- Acuerdos de licencia: Obtención de la autorización legal para la fabricación, uso y/o explotación comercial de tecnología y conocimiento protegidos mediante derechos de propiedad industrial e intelectual. Puede tratarse de una licencia de patente, diseño, software, marca y/o know-how (secreto industrial); o una franquicia (licencia conjunta de todos los derechos de un negocio: tecnología, marca, contactos comerciales, procedimientos de trabajo). 
- Cooperación tecnológica: Colaboración en el marco de un proyecto de investigación y desarrollo (I+D) para generar nuevas tecnologías, productos o procesos, bien sea bajo contrato (el receptor subcontrata al proveedor), o como un proyecto conjunto en pequeños o grandes consorcios (la tecnología es co-desarrollada entre las partes a través de una relación de socios).

- Asistencia técnica y servicios: Prestación de asesoramiento técnico y/o servicios especializados fuera de lo contemplado por derechos de propiedad o secreto industrial, bajo la forma de asistencia técnica (asesoramiento, consultoría, ingeniería, estudios, apoyo tecnológico); servicios especializados (ensayos, análisis, certificaciones); formación en áreas clave; o subcontratación industrial (fabricación, suministro de componentes).

- Movilidad de personal: Incorporación de personal experto o conocedor de áreas científicas o técnicas, de manera permanente (contratación) o temporal (estancia, intercambio).

- Creación de empresas: Para la explotación comercial de una tecnología o conocimiento, donde la tecnología o conocimiento base de la empresa es generalmente el resultado de un proceso de I+D.

- Alianzas tecnológicas: Colaboración entre las partes para compartir activos, riesgos, costes, beneficios, capacidades o recursos en torno al desarrollo y/o explotación de tecnología y conocimiento. Puede consistir en la creación de una empresa a medida (joint venture, unión temporal de empresas); la colaboración para la transferencia puntual de capacidades entre los socios; o un consorcio de I+D colaborativa (participación en programas públicos de I+D).

- Adquisiciones y fusiones: Fusión con (o adquisición de) una organización intensiva en tecnología y conocimiento. La inversión contempla la transferencia de una empresa completa, incluyendo sus activos tangibles e intangibles (intelectuales, humanos y comerciales).

- Compra/venta de bienes y equipos: Adquisición de activos tangibles intensivos en capital disponibles comercialmente, puede tratarse de bienes de equipos o de soluciones TIC (tecnologías de la información y comunicación) en forma de software o hardware comercial. La tecnología va oculta en forma de conocimiento y/o derechos de propiedad y la transferencia se produce con la mera compra o adquisición del activo. El contrato de compra-venta suele incluir (automáticamente) la concesión de las licencias no exclusivas de uso de la tecnología incorporada. La compra de bienes de equipo puede ser complementada con la prestación de servicios de carácter técnico u otros (instalaciones, formación).

En síntesis en cuanto a los mecanismos de transferencia de tecnología y conocimiento, se observa que no existen instrumentos que permitan determinar la forma en que debe llevarse a cabo el proceso, basado en la identificación de necesidades tecnológicas propias de los proveedores. En este sentido, la primera necesidad es la de transferir genéticamente embriones que permitan la obtención de crías con mayor adaptabilidad a las condiciones ambientales y los requerimientos del proveedor, es decir, si necesita solo animales de color blanco, se trabajará con líneas genéticas que permitan esta característica por ejemplo la cebú, que además del color, su rusticidad le permite mayor adaptabilidad a las altas temperaturas de la zona.

Este proceso puede extenderse a toda la región del departamento del Atlántico, dado que Barranquilla no es el único municipio que provee animales; todas sus cabeceras municipales participan en este proyecto, dada la privilegiada posición geográfica dotada de un puerto marítimo con condiciones aptas para el desarrollo del sector exportador de ganado bovino, que promueve un alcance de gran impacto económico para la economía agropecuaria colombiana.Para los efectos de la presente investigación, se toma en cuenta la clasificación propuesta por González-Sabater (2011), por considerar que abarca elementos característicos que se manifiestan en el contexto de las organizaciones del sector ganadero, en cuanto a sus procesos de 
transferencia del conocimiento y adquisición de tecnologías. Debido a todo lo expuesto en este trabajo se busca determinar los mecanismos de transferencia de tecnología y conocimiento en las empresas exportadoras de ganado bovino de Barranquilla, Colombia.

\section{Metodologia}

Atendiendo al grado de conocimiento del objeto de estudio la presente investigación se considera descriptiva y de pronóstico. Al respecto, Ríos (2017) señala que esta investigación corresponde al "segundo nivel de conocimiento, busca encontrar las características, comportamiento y propiedades del objeto de estudio, ya sea en el presente o en el futuro, en este último caso se denomina de pronóstico" (p. 81).

Para el presente trabajo, el diseño se considera de campo, Arias (2012), indica que la investigación de campo consiste en la recolección de datos directamente de la realidad donde objeto de estudio, sin manipular variable alguna. Al mismo tiempo, se considera un diseño no experimental transeccional (transversal), ya que se recolectan los datos en un solo momento y en un lapso de tiempo específico.

\subsection{Población y muestra}

La población corresponde al conjunto de especialistas de la Asociación de Ganaderos de la Costa Norte (ASOGANORTE) vinculados al sector bovino; así como profesionales con experticia en Buenas Practicas de Ganadería (BPG) en empresas exportadoras que operan desde la ciudad de Barranquilla, Departamento del Atlántico.

Por su parte, la muestra, su selección responde a un muestreo no probabilístico intencional, dado que el investigador "escoge sus unidades no en forma fortuita sino completamente arbitraria, designando a cada unidad según características que para el investigador resulten de relevancia” (López, 2011, p. 170).

Es importante acotar, que dicha selección obedece a los siguientes criterios: (1) expertos en actividades de exportación de ganado bovino; y (2) actores empresariales que, para el momento de realizar la recolección de datos, se encuentren ejecutando tales actividades, o supervisando el cumplimiento de las BPG. Es así como, la muestra se compone de un total de quince (15) sujetos, tal como se observa en la Tabla 1

Tabla 1

Muestra del estudio

\begin{tabular}{|c|c|c|}
\hline Organismo & Criterio de inclusión considerado & Cantidad \\
\hline ASOGANORTE & Criterios (1) y (2) & 12 \\
\hline SGS & Criterio (3) & 3 \\
\hline & Total de la muestra & 15 \\
\hline
\end{tabular}

\subsection{Técnicas e instrumentos de recolección de datos}

La técnica de recolección de datos utilizada consiste en la observación mediante encuesta. Palella \& Martins (2012), señalan que es una técnica destinada a captar datos de varias personas cuyas respuestas importan al investigador. En tal sentido, se aplicará la encuesta para la recolección de los datos respecto a la variable Estrategia de Transferencia de Tecnología y Conocimiento. Por otro lado, como instrumento de recolección de datos se emplea un cuestionario. Este se define, según López (2011, p. 193), como "un instrumento que agrupa una serie de preguntas relativas a un evento, situación o temática sobre la cual el investigador desea obtener información". De manera que, se diseñó un cuestionario, bajo una escala tipo Likert construida a partir de cinco (5) alternativas de respuestas, a saber: Siempre; Casi siempre; Algunas veces, Casi nunca y Nunca, valoradas por medio de una puntuación entre cinco y uno, respectivamente. 


\subsection{Técnicas de análisis de resultados}

Los datos fueron tabulados, expresados en valores de frecuencia absoluta y relativa para cada uno de los indicadores propios de cada dimensión estudiada. Por ello, se procede bajo un análisis estadístico descriptivo, el cual para, Arias (2012), consiste en realizar un análisis específico de cada pregunta del cuestionario, para luego realizar un análisis general en relación con la operacionalización de la variable. Se llevó a cabo un análisis de media aritmética para las diferentes dimensiones de la variable, cuya interpretación corresponde a una valoración de acuerdo con las categorías listadas en la Tabla 2.

Tabla 2

Baremo para la interpretación del promedio

\begin{tabular}{|c|c|c|}
\hline Rango & Valor & Categoría \\
\hline $1.00-1.80$ & 1 & Muy baja presencia (MBP) \\
\hline $1.81-2.60$ & 2 & Baja presencia (BP) \\
\hline $2.61-3.40$ & 3 & Mediana presencia (MP) \\
\hline $3.41-4.20$ & 4 & Alta presencia (AP) \\
\hline $4.21-5.00$ & 5 & Muy alta presencia (MAP) \\
\hline
\end{tabular}

\section{Resultados y Discusion}

Como se muestra en la Tabla 3 los diferentes indicadores de la Dimensión Mecanismos de transferencia, corresponden a los siguientes: (1) Acuerdos de licencia; (2) Cooperación tecnológica; (3) Asistencia técnica y servicios; (4) Movilidad de personal; (5) Creación de empresas; (6) Alianzas tecnológicas; (7) Adquisiciones y fusiones; y (8) Compra/venta de bienes y equipos, cuyos resultados fueron analizados y contrastados con las teorías que soportan el estudio.

Tabla 3

Mecanismos de transferencia

\begin{tabular}{|c|c|c|c|c|c|c|c|c|c|c|c|c|c|}
\hline \multirow{2}{*}{ Indicadores } & \multicolumn{2}{|c|}{$S$} & \multicolumn{2}{|c|}{ CS } & \multicolumn{2}{|c|}{ AV } & \multicolumn{2}{|c|}{$\mathrm{CN}$} & \multicolumn{2}{|c|}{$\mathbf{N}$} & \multicolumn{3}{|c|}{ Media } \\
\hline & $\mathrm{Fa}$ & $\%$ & $\mathrm{Fa}$ & $\%$ & $\mathrm{Fa}$ & $\%$ & $\mathrm{Fa}$ & $\%$ & $\mathrm{Fa}$ & $\%$ & Rango & Valor & Categoría \\
\hline $\begin{array}{l}\text { Acuerdos de } \\
\text { licencia }\end{array}$ & 32 & 71 & 8 & 18 & 5 & 11 & 0 & 0 & 0 & 0 & 4,60 & 5 & MAP \\
\hline $\begin{array}{l}\text { Cooperación } \\
\text { tecnológica }\end{array}$ & 6 & 13 & 13 & 29 & 19 & 42 & 7 & 16 & 0 & 0 & 3,40 & 3 & $\mathrm{MP}$ \\
\hline $\begin{array}{l}\text { Asistencia técnica y } \\
\text { servicios }\end{array}$ & 9 & 20 & 12 & 27 & 6 & 13 & 18 & 40 & 0 & 0 & 3,27 & 3 & $\mathrm{MP}$ \\
\hline $\begin{array}{l}\text { Movilidad de } \\
\text { personal }\end{array}$ & 6 & 13 & 11 & 24 & 20 & 44 & 8 & 19 & 0 & 0 & 3,33 & 3 & $\mathrm{MP}$ \\
\hline $\begin{array}{l}\text { Creación de } \\
\text { empresas }\end{array}$ & 4 & 9 & 11 & 24 & 14 & 31 & 16 & 36 & 0 & 0 & 3,07 & 3 & MP \\
\hline $\begin{array}{l}\text { Alianzas } \\
\text { tecnológicas }\end{array}$ & 6 & 13 & 11 & 24 & 20 & 44 & 8 & 19 & 0 & 0 & 3,33 & 3 & MP \\
\hline $\begin{array}{l}\text { Adquisiciones y } \\
\text { fusiones }\end{array}$ & 2 & 7 & 11 & 37 & 3 & 10 & 14 & 46 & 0 & 0 & 3,03 & 3 & $\mathrm{MP}$ \\
\hline $\begin{array}{l}\text { Compra/venta de } \\
\text { bienes y equipos }\end{array}$ & 6 & 13 & 15 & 33 & 8 & 18 & 16 & 36 & 0 & 0 & 3,24 & 3 & $\mathrm{MP}$ \\
\hline Media General & & & & & & & & & & & 3.43 & 3 & MP \\
\hline
\end{tabular}

Fuente: Elaboración propia

En primer lugar se presenta el Indicador Acuerdos de licencia donde la mayoría de los encuestados se ubicó en la escala de respuesta Siempre con un $71 \%$, mientras que para la alternativa Casi Siempre concentra un $18 \%$, y la opción Algunas veces con el $11 \%$ restante, sin respuestas para las opciones Casi nunca y Nunca. En síntesis, el 
indicador analizado se ubica dentro de la categoría Muy Alta Presencia (MAP) de acuerdo al baremo, evidenciando un 4,60 de la media aritmética, siendo el más alto de esta dimensión.

Las respuestas suministradas por los encuestados aportan al respecto que el principal mecanismo de licenciamiento corresponde a la protección de los derechos de propiedad intelectual e industrial de la tecnología y el conocimiento transferidos. En este orden de ideas, Arenas (2012) y Solis et al. (2020) afirman que esta forma de transferencia puede ser exclusiva o no. Citando a Rogers (2000), lo considera "como un permiso o los derechos para utilizar cierto producto, diseño o proceso. Usualmente las licencias conforman un pago por el intercambio de información" (p. 19).

En cuanto al indicador Cooperación tecnológica, se tiene que un 13\% respondió la alternativa Siempre; un 16\% se inclinó por responder Casi nunca; mientras que el 29\% señaló Casi siempre; siendo la mayor frecuencia correspondiente a la opción Algunas veces con un 42\%, sin respuestas registradas para Nunca. Éste indicador obtuvo una media ponderada de 3,40 ubicándose en la categoría de Mediana Presencia (MP) de acuerdo al baremo establecido.

Estos resultados manifiestan que es poco frecuente que en el sector exportador de ganado en Barranquilla se lleven a cabo procesos de transferencia basados en proyectos conjuntos colaborativos en la generación de nuevo conocimiento tecnológico. Siguiendo con González-Sabater (2011) y Simon et al. (2020), este mecanismo es la "colaboración en el marco de un proyecto de investigación y desarrollo (I+D) para generar nuevas tecnologías, productos o procesos" (p. 48).

Seguidamente, para el indicador Asistencia técnica y servicios, se registra un $40 \%$ de respuestas para la opción Casi nunca como la mayor frecuencia relativa obtenida, seguida de un $27 \%$ para la alternativa Casi siempre, $20 \%$ para Siempre y el $13 \%$ restante Algunas veces, sin respuestas ofrecidas para la opción Nunca. La media aritmética obtenida para el indicador es de 3,27, lo que de acuerdo con el baremo representa una Mediana Presencia (MP) del mismo en la muestra estudiada.

Estos resultados muestran debilidades en el indicador, en cuanto al poco asesoramiento técnico por parte del proveedor, más que todo en la prestación de servicios especializados en áreas clave del negocio. GonzálezSabater (2011, p. 48) y Leyva et al. (2020) explican que este mecanismo consiste en la "prestación de asesoramiento técnico y/o servicios especializados fuera de lo contemplado por derechos de propiedad o secreto industrial".

En cuanto al indicador Movilidad de personal, se muestra un 44\% de encuestados a favor de la opción Algunas veces, como la de mayor frecuencia relativa, seguida de un $24 \%$ para la opción Casi siempre y otro $19 \%$ Casi nunca, siendo el restante $13 \%$ quienes seleccionaron Siempre, sin respuestas registradas para Nunca. Estos valores colocan al indicador con una media aritmética de 3,33, en la categoría Mediana Presencia (MP), siendo a su vez la más alta entre los indicadores de la dimensión considerada.

Es así como los resultados expresan que principalmente, el personal movilizado obedece a la contratación permanente de personal experto, lo cual se corresponde con los planteamientos de Delgado (2017) y Meza et al. (2020), quienes sostiene que este mecanismo de transferencia de conocimiento "brinda oportunidades de aprendizaje y cooperación; además estimula la creación de nuevo conocimiento con el cual se puede llegar a innovar en la organización" (p. 31).

Seguidamente, para el indicador Creación de empresas, se registra un 36\% de respuestas para la opción Casi nunca como la mayor frecuencia relativa obtenida, seguida de un 31\% para la alternativa Algunas veces, $24 \%$ para Casi siempre y el 9\% restante Siempre, sin respuestas ofrecidas para la opción Nunca. La media aritmética 
obtenida para el indicador es de 3,07, lo que de acuerdo con el baremo representa una Mediana Presencia (MP) del mismo en la muestra estudiada.

Bajo esta premisa, las empresas exportadoras de ganado en Barranquilla consideran muy poco este indicador como mecanismo de transferencia, toda vez que no se promueven emprendimientos basados en resultados de investigaciones académicas para la explotación comercial de una tecnología obtenida de un proceso de I+D. Esta situación se contrapone a lo expresado por Pérez (2012), quien señala que "una institución puede externalizar el desarrollo y explotación comercial de una tecnología a través de la creación de una empresa independiente, pero vinculada a ella mediante participaciones de capital u otros nexos" (p. 8). A esto se le conoce como empresa de base tecnológica, o spin-off.

Por su parte, se tiene el indicador Alianzas tecnológicas, para el que un $44 \%$ de encuestados a favor de la opción Algunas veces, como la de mayor frecuencia relativa, seguida de un $24 \%$ para la opción Casi siempre y otro $19 \%$ Casi nunca, siendo el restante $13 \%$ quienes seleccionaron Siempre, sin respuestas registradas para Nunca. Estos valores colocan al indicador con una media aritmética de 3,33, en la categoría Mediana Presencia (MP).

Es así como los resultados apoyan las afirmaciones de Pérez (2012) quien afirma que una alianza tecnológica "consiste en explotar comercialmente una tecnología en conjunto con otras empresas, siendo el objetivo de esto conseguir un objetivo empresarial, normalmente delimitado, basado en reducir costes y riesgos" (p. 8). De esta manera, las empresas consultadas consideran los Joint Venture de I+D como mecanismo en el que se comparten costos para recibir beneficios mutuos de la comercialización, así como de los recursos de los que ambas disponen para la explotación del conocimiento y/o tecnología objeto de transferencia.

Seguidamente, para el indicador Adquisiciones y fusiones, se registra un $46 \%$ de respuestas para la opción Casi nunca como la mayor frecuencia relativa obtenida, seguida de un $37 \%$ para la alternativa Casi siempre, $10 \%$ para Algunas veces y el 7\% restante Siempre, sin respuestas ofrecidas para la opción Nunca. La media aritmética obtenida para el indicador es de 3,03, lo que de acuerdo con el baremo representa una Mediana Presencia (MP) del mismo en la muestra estudiada.

Al respecto, González-Sabater (2011, p. 48) señala que bajo este mecanismo, "la inversión contempla la transferencia de una empresa completa, incluyendo todos sus activos tangibles e intangibles (intelectuales, humanos y comerciales)". De esta manera, los resultados evidencian que, en las empresas consultadas, no se ha contemplado esta situación.

Finalmente, el análisis del indicador Compra/venta de bienes y equipos, expresa que el 36\% optó por la alternativa Casi nunca, correspondiéndole la valoración más alta registrada, además de las opciones Casi siempre con un $33 \%$, Algunas veces se presenta con un $18 \%$, Siempre con un $13 \%$, y sin respuestas para la alternativa Nunca. El análisis de media muestra un resultado de 3,24 por lo que el indicador se manifiesta con Mediana Presencia (MP) según baremo.

Los resultados evidencian que, bajo ciertas condiciones, las empresas recurren a la adquisición de activos tangibles disponibles comercialmente, con limitaciones en la prestación de servicio técnico complementario a la compra. Al respecto, Pérez (2012) y Vincent (2020) afirman que en la venta de tecnología, su propiedad "se traspasa totalmente a la empresa que la compra. Suele darse en los casos en que la tecnología generada diverge del negocio de la empresa que la ha generado y/o los costes de desarrollarla comercialmente son excesivamente altos" (p. 8). 
En síntesis, el análisis realizado a partir del cálculo de la media general para la dimensión Mecanismos de transferencia fue de 3.43, correspondiendo a la categoría de Mediana Presencia (MP), según el baremo elaborado. Este análisis se apoya en el hecho de que todos los indicadores correspondientes a la dimensión en cuestión se ubicaron en la misma categoría, excepto el indicador Acuerdos de Licencia que se posicionó con una Muy Alta Presencia.

De acuerdo a las bases teóricas consideradas, y dando respuesta al segundo objetivo específico, se ratifica la importancia que reviste la dimensión analizada, de manera particular los planteamientos de Pérez (2012) y Fernández et al. (2020) quienes destaca que "la comercialización de I+D+i está basada en los mecanismos de transferencia de tecnología", siendo este proceso quien "acerca el conocimiento a las empresas para generar un impacto en el mercado y en la sociedad, que revierta en riqueza y competitividad" (p. 6).

\section{Conclusiones}

Expuestos y analizados los resultados, se concluye que, dados los resultados aportados por el análisis estadístico descriptivo realizado, la más alta valoración corresponde a los acuerdos de licencia, siendo estos el principal mecanismo de transferencia acometido por las empresas estudiadas. En consecuencia, se manifiesta el mediano interés en implementar otros mecanismos de transferencia, muchos de los cuales tienen cabida en ambientes de vinculación entre el sector académico y el empresarial, donde la transferencia se expresa como la movilización de la tecnología desde los centros generadores del conocimiento hacia las empresas que requieren su uso y explotación, siendo en este caso, una transferencia horizontal o intersectorial. En el caso de estudio, la transferencia es vertical o intrasectorial, producida dentro de un mismo sector de la economía, y dado el tipo de contraprestación recibida de acuerdo con los resultados del estudio, resulta válida la consideración de que los acuerdos de licencia sean el mecanismo principal de transferencia de tecnología y conocimiento en las empresas exportadoras de ganado bovino.

\section{Referencias bibliográficas}

Arenas, J. (2012). Propuesta de metodología de un proceso de transferencia tecnológica de la universidad a la empresa. (Trabajo de maestría). Pontificia Universidad Católica del Perú, Lima, Perú.

Arias, F (2012). El proyecto de investigación científica: Introducción a la metodología científica. Caracas, Venezuela: Editorial Episteme.

Arias, L. M. B., \& Segura, F. S. (2020). La gestión del conocimiento para la innovación y el desarrollo rural: experiencias desde Costa Rica. Yulök Revista de Innovación Académica, 4(1).

Bornachera, K. P., Juvinao, D. D. L., \& Jaramillo, A. H. (2020). Transferencia tecnológica para la producción limpia en la minería de materiales aluviales en La Guajira, Colombia. Investigación e Innovación en Ingenierías, 8(1), 6-20.

Casar, J (2004) Tecnología de la información y las comunicaciones. Recuperado de http://www.upm.es/consejosocial/tic.pdf

Delgado, S. (2017). Desarrollo de un modelo de transferencia del conocimiento para centros de investigación. Estudio de caso: Centro de estudios avanzados en niñez y juventud alianza CINDE - Universidad de Manizales. (Trabajo de maestría). Universidad Nacional de Colombia, Manizales, Colombia. 
Fernández, Y. O., Fernández, A. V., Suárez, E. G., Villegas, D. A., Gamboa, J. N., \& Echevarria, T. I. L. (2020). Gestión del conocimiento y tecnologías de la información y comunicación (TICs) en estudiantes de ingeniería mecánica. Apuntes Universitarios, 10(1), 77-88.

González-Sabater, J. (2011). Manual de transferencia de tecnología y conocimiento. Alicante, España: Instituto de transferencia de tecnología y conocimiento.

Lee, J. (2003). Technology transfer between university research centers and industry in Singapore. Technovation, 24 (1): 433-442.

Leyva-Morales, C., Sabido-Ocampo, H., \& Vela-Manzanilla, R. (2020). Elementos de Relevancia sobre la Evaluación del Programa Fomento Ganadero en Yucatán, México, 2001-2013. Revista Salud y Administración, 7(19), 23-38.

Linzer, G. A. (2008). Devenir de la generación y transferencia de conocimientos en el Instituto Nacional de Tecnología Agropecuaria de la Argentina. Arbor, 184(732), 701-717.

López, E. (2011). Metodología de la investigación: Guía instruccional. Caracas: Universidad Nacional Abierta.

Mango, A., Sabourin, E., Samper, M., Sotomayor, O., Basualdo, A., Petri, G., ... \& Thornton, R. (2016). Cosechando resultados: Paraguay: logros significativos en 2015. In World Forestry Congress1318-23 October, 2009Buenos Aires, Argentina (No. IICA E14). IICA, San José (Costa Rica).

Meza-Sepulveda, D. C., Quintero-Saavedra, J. I., Zartha-Sossa, J. W., \& Hernández-Zarta, R. (2020). Estudio de prospectiva del sector cacao al año 2032 como base de programas de capacitación universitaria en el sector agroindustrial. Aplicación del método Delphi. Información tecnológica, 31(3), 219-230.

Ortuño, A. D. (2007). DETERMINACION DE PRIORIDADES DE INVESTIGACIQN Y TRANSFERENCIA TECNOLOGICA DE LA FMVZ" DR. NORBERTO TREVIÑO ZAPATA". CienciaUAT, 2(1), 63-66.

Palella, S. \& Martins, F. (2012). Metodología de la investigación cuantitativa. Caracas, Venezuela: FEDUPEL.

Pérez, A. (2012). Hacia una nueva cultura empresarial: la transferencia de tecnología y de conocimiento. 3ciencias Revista de investigación. 1-16. Recuperado de https://www.3ciencias.com/wpcontent/uploads/2012/10/ARTICULO-ANA.pdf

Reyes, J. (2010). Transferencia tecnológica en institutos universitarios de tecnología. Revista Electrónica Facultad de Ingeniería UVM, 4(2): 280-296.

Ríos, R. (2017). Metodología para la investigación y redacción. Málaga, España: Servicios Académicos Intercontinentales S.L.

Rogers, Et. (2000). Lessons learned about technology transfer. Technovation, 21 (1), 253-261.

Simón-Díaz, B. F., Robles, G. G., \& Ponce, W. C. C. (2020). Modelo de gestión de conocimiento para el programa nacional de alimentación escolar Qali Warma. Investigación Valdizana, 14(1), 15-21.

Solís Lima, A., Ovando, C., Olivera Pérez, E., \& Rodríguez Lozada, M. Á. (2020). Desempeño de una Oficina de Transferencia de Tecnología en el contexto de gestión de patentes: Estudio de caso de la OTT de la Benemérita Universidad Autónoma de Puebla. Nova scientia, 12(24).

Trigo, E. J., \& Elverdin, P. (2020). Los sistemas de investigación y transferencia de tecnología agropecuaria de América Latina y el Caribe en el marco de los nuevos escenarios de ciencia y tecnología. Revista Compromiso Social, (3), 116-127. 
Vincent, S. (2020). Models of Innovation Systems for Utilization of Traditional Knowledge in Farm Livestock. Revista Cientifica-Facultad de Ciencias Veterinarias, 30(5).

Zarazúa, J. A., Solleiro, J. L., Altamirano Cárdenas, R., Castañón Ibarra, R., \& Rendón Medel, R. (2009). Esquemas de innovación tecnológica y su transferencia en las agroempresas frutícolas del estado de Michoacán. Estudios sociales (Hermosillo, Son.), 17(34), 37-71.

Esta obra está bajo una Licencia Creative Commons Attribución-NoCommercial 4.0 International

(c) BYY-NO 